RESONANCIAS

(reseñas)

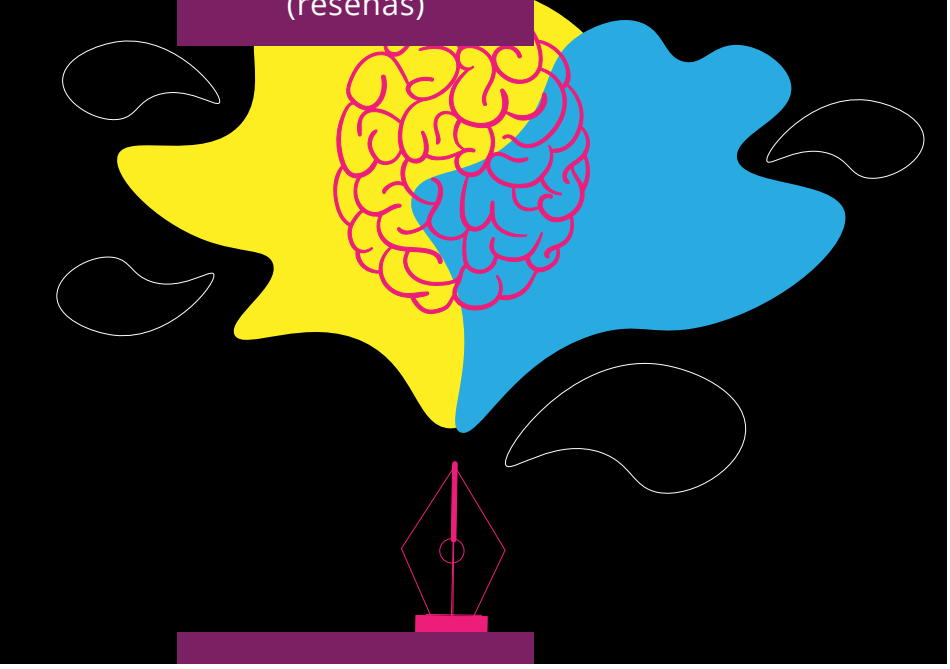

FIGURAS REVISTA ACADÉMICA DE INVESTIGACIÓN ISSN 2683-2917

Vol. 2, núm. 3, julio-octubre 2021

https://doi.org/10.22201/fesa.figuras.2021.2.3

Esta obra está bajo una licencia Creative Commons Atribución-NoComercialCompartirlgual 4.0 Internacional

\section{Vinculación de experiencias didácticas con el entorno laboral a través del pensamiento creativo en la Licenciatura en Diseño Gráfico}

\section{Linking Didactic Experiences with the Work Environment Through Creative Thinking in the Graphic Design Degree Program}

https://doi.org/10.22201/fesa.figuras.2021.2.3.170

Elvia Mónica Rodríguez-Alonso

Universidad Nacional Autónoma de México.

Facultad de Estudios Superiores Acatlán
En esta reseña didáctica exponemos el proceso que se siguió en la materia Campaña Publicitaria de la Licenciatura en Diseño Gráfico de la Facultad de Estudios Superiores (FES) Acatlán en el año 2017. En esa ocasión se invitó a participar a alumnos de $9^{\circ}$ semestre de la FEs y de la Universidad La Salle, y se logró comprobar el éxito de dicho proceso con los resultados obtenidos.

\section{Introducción}

La empresa UNIFIN ${ }^{\circledR 1}$ invitó a la Universidad La Salle y a la FEs Acatlán (UNAM), a través de una convocatoria el 30 de agosto del 2016, a participar en el diseño de su campaña publicitaria.

La dinámica del concurso inspiró a los alumnos a colaborar e involucrarse en el proceso laboral para la realización de campañas publicitarias para un cliente real, esto hizo que su experiencia didáctica se fortaleciera durante el semestre.

De la FEs Acatlán participaron alumnos de la materia Campaña Publicitaria de la Licenciatura en Diseño gráfico, tanto del turno matutino como vespertino.

Se organizó una reunión entre el cliente y los estudiantes interesados en desarrollar ese proyecto durante el semestre 2017-1. El objetivo de esa sesión fue dar a conocer las bases de la convocatoria, las características de la campaña que el cliente requería (brief) y que los alumnos aprendieran la forma en que se desarrolla la explicación del brief al inicio de un proyecto de este tipo para, posteriormente, comenzar con el proceso de ejecución de las propuestas para la campaña que se implementaría en el 2017.

1 unifin.com.mx 2020. Todos los derechos de marca son reservados por el mismo cliente. 


\section{Objetivos del aprendizaje}

1. Identificar los requerimientos principales de comunicación a través del desarrollo de una investigación de mercados.

2. Plantear un modelo estratégico creativo que determine la solución de comunicación en los diferentes medios, principalmente: Tv (abierta y de paga), espectacular (norte, centro y sur de las ciudades principales), revistas, periódicos, eventos deportivos (golf y Fórmula 1) y redes sociales.

\section{Contenidos del programa de la materia}

La selección y organización de contenidos se fue dando durante las clases del semestre; el alumno fue adquiriendo conceptos relacionados con la publicidad y su aplicación en el diseño gráfico. Estos contenidos van asociados con las bases teóricas, metodológicas y creativas para poder iniciar su proceso creativo para el desarrollo de la propuesta publicitaria.

Los contenidos estuvieron estrechamente relacionados con el programa de la materia: conceptos y clasificación de publicidad, mercadotecnia, sistemas de investigación de mercados, agencias de publicidad, el brief (solicitud del cliente), plataforma creativa con estrategias y procesos, metodologías, diseño del mensaje, canales de comunicación, presupuestos y breve introducción de cómo es la medición de resultados cuando la campaña ya está implementada en los medios seleccionados.

\section{Materiales didácticos}

Durante la primera fase se utilizaron materiales básicos como papel, post-it, plumones, entre otros para el desarrollo de sus primeras ideas, además del acompañamiento del software de diseño para ir realizando los avances de lo que están ejecutando para su proyecto.

\section{Contexto}

UNIFIN $^{\circledR}$ es una empresa financiera totalmente mexicana, con más de veinte años de experiencia. La finalidad de la campaña fue difundir a nivel nacional los productos principales de su portafolio de servicios:

- Leasing, dirigido principalmente a las pequeñas y medianas empresas (PYMES), personas morales y personas físicas con actividad empresarial.

- Factoring, referente al factoraje financiero para el apoyo a proveedores de cadenas medianas y grandes que facturan más de 6 millones de pesos anuales. Principalmente para el sector industrial, hipermercados y grandes cadenas.

- Credit, básicamente enfocado al crédito automotriz para brindar financiamiento de vehículos nuevos y seminuevos con una tasa anual fija. Su pretensión es dirigirse principalmente a los millennials.

- Insurance, su enfoque primordial son los seguros y las fianzas. Cuentan con asesoramiento a las empresas y a las personas con soluciones integrales adecuadas a sus necesidades: proporcionan siempre las mejores alternativas en costo-beneficio para garantizar la seguridad en vida, salud y contra cualquier siniestro que amparen las pólizas.

- Fórmula 1, patrocinadores oficiales con Gaby López y en su momento con Esteban Gutiérrez.

Su misión es "ofrecer la más alta calidad en servicios financieros hechos a la medida de cada uno de nuestros clientes para poder satisfacer necesidades". ${ }^{2}$ Cuentan con oficinas en Cancún, CDMX, Chihuahua, Guanajuato, Jalisco, Mérida, Nuevo León, Puebla, Querétaro, Sonora, Toluca y Veracruz.

La materia de Campaña Publicitaria debe cumplir su objetivo durante el semestre, que consiste en

2 unifin.com.mx Revisado el 9 de junio, 2021. 
el aprendizaje de conceptos, metodologías y herramientas tradicionales y digitales para el desarrollo de campañas publicitarias para cualquier sector comercial. Los objetivos principales que el cliente pretende obtener con la campaña son: generar otros clientes potenciales, aumentar las ventas, fortalecer su imagen corporativa como líder en el mercado financiero y generar una estrategia alineada a su comunicación en las redes sociales.

\section{Desarrollo creativo}

Algunos aprendizajes que atienden y adquieren los alumnos -dentro de este proceso de pensamiento de diseño- están relacionados con las siguientes actividades durante el semestre, entre ellas se encuentran: proceso de briefing, estructurar un equipo de trabajo, planificación y administración del proyecto de diseño, establecer costos, documentación y sistemas de control del proyecto, recomendaciones de diseño final, implementación de las soluciones y evolución de los proyectos de diseño.

Se describen tres fases para el desarrollo de las propuestas de campaña publicitaria; en cada una de ellas se explicará la metodología y aspectos que se consideraron para su ejecución. La primera es el desarrollo creativo, donde se describe la formación metodológica que emplearon los alumnos para su proyecto. La segunda, situada en la propuesta de solución, es donde las primeras propuestas de campañas presentadas por los alumnos son seleccionadas para la decisión final del cliente. Finalmente, en la tercera fase se describe la experiencia de los alumnos al conocer el resultado de ser los ganadores y aprenden la relación directa con el cliente para el desarrollo de los diseños finales y la entrega total de la campaña para su futura implementación en los medios de comunicación seleccionados por el cliente.
Primera fase: Propuesta creativa de la campaña

La propuesta se elaboró utilizando la metodología de Design Thinking o pensamiento de diseño, que consiste en analizar una situación con el objetivo de encontrar la solución efectiva de comunicación que se le solicita al diseñador gráfico.

Esta metodología tiene su origen en la Universidad de Stanford de California. ${ }^{3}$ Durante los años 70 la consultora de diseño $\mathrm{IDEO}^{4}$ fue la primera en aplicarla en proyectos comerciales y, hasta la fecha, sigue siendo un referente en su uso y en innovación.

La característica principal de dicha metodología es centrarse en el usuario final que recibirá la solución, a partir del estudio de la situación a resolver. Así se ofrece una propuesta de $360^{\circ}$, es decir, que utilice todos los medios a disposición para comunicar el prototipo final en el entorno solicitado.

El Design Thinking hace referencia a los procesos estratégicos, cognitivos y operativos que se desarrollan a través de conceptos relacionados con las diferentes áreas del diseño. Este tipo de pensamiento de diseño también está asociado con aspectos de innovación de productos y servicios en diversos contextos empresariales y sociales.

En nuestro caso, UNIFIN ${ }^{\circledR}$ comprende un entorno totalmente financiero en donde la metodología se vuelve muy dinámica para resolver una situación planteada y posicionar el portafolio de negocio del que dispone la marca.

El proceso que siguen los alumnos para hacer sus propuestas parte de la investigación del mercado, segmentación del target (perfil del usuario final), análisis de los medios actualmente utilizados, análisis de la campaña vigente, brainstorming (lluvia de

3 Sitio de la Universidad de Stanford para mayor información: www.stanford.edu

4 Sitio de IDEO para mayor información: www.ideo.com 
ideas), propuesta de valor de marca, creativo y elaboración de propuestas, con la finalidad de realizar la presentación de la campaña que enviarán al cliente para su participación en dicha convocatoria.

\section{Propuesta de solución}

\section{Segunda fase: Experiencia de los alumnos} durante la revisión y selección de propuestas

Independientemente de la participación de otras clases de campaña publicitaria de la Licenciatura en Diseño Gráfico, tanto del turno matutino como del vespertino, y tomando en consideración los grupos que estaban a mi cargo, el 1952 (11 equipos) y el 1953 (de 9), se realizó una implicación positiva por parte de la comunidad de $9^{\circ}$ semestre.

La entrega formal de las propuestas de dicha campaña para la convocatoria fue el 9 de diciembre de 2016, con 17 proyectos finalistas previamente seleccionados que cubrían los requerimientos y la calidad gráfica para seguir participando: 10 del grupo 1952, 5 del 1954 y 2 del 1901. UNIFIN ${ }^{\circledR}$, por su parte, se dio a la tarea de revisar las propuestas entregadas a través de un comité interno seleccionado por el cliente, conformado por personal de las áreas de Dirección Comercial, Dirección de Mercadotecnia y Diseño y Compras.

Tercera fase: Resultados y desarrollo de diseños para implementación

La figura 1 refleja en resumen todo el proceso que se llevó a cabo para hacer las propuestas publicitarias para la convocatoria expuesta por el cliente UNIFIN ${ }^{\circledR}$, previamente explicado a detalle.

El 9 de enero de 2017 el equipo de mercadotecnia de UNIFIN ${ }^{\circledR}$ notificó oficialmente ganadora a la FES Acatlán con la campaña "Armar" del equipo $\mathrm{M}+\mathrm{A}$, perteneciente a los alumnos Alberto Crespo Picazo, Mariana Hernández Canchola, Juan Antonio Torres César y Moisés Pureco Vázquez. Además, la FEs tuvo la grata sorpresa de recibir también el zer lugar.
Convocatoria

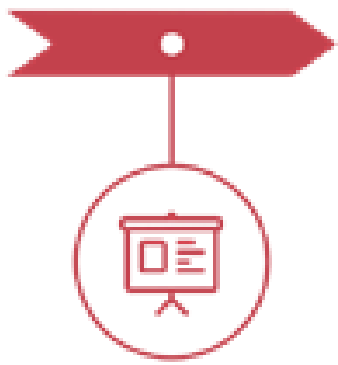

La Salle vs FES Acatlán

Lanzamiento formal el 30 de agosto del 2016
Brief

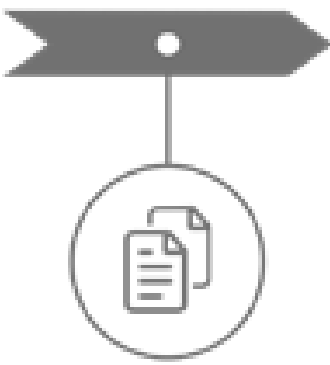

FES ACATLAN

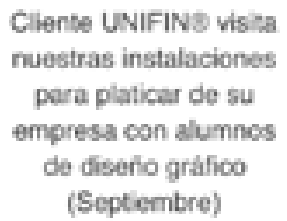

Participación

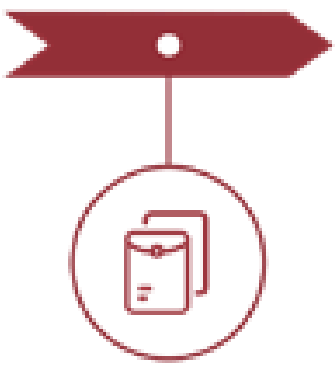

PROYECTOS

Inscripcion de proyectos de campara (matutino \& vesperino)
Selección

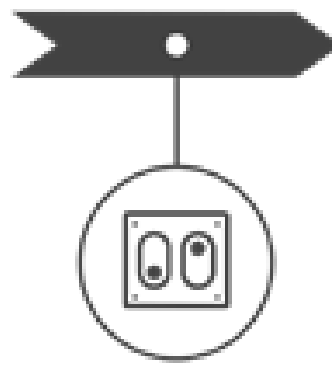

FINALISTAS

UNIFIN selecciona los proyectos para tomar la decisión final (Noviembre)
Resultado

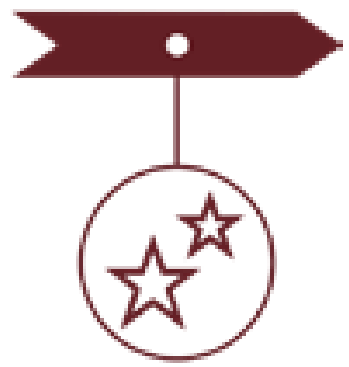

1er \& 3er lugar

Nobificación de ganadores y preparación de artes para entrega final

Figura 1. Esquema realizado por Elvia Mónica Rodríguez-Alonso, 2019. 
Los alumnos ganadores, al conocer el resultado, se llenaron de alegría. Se les indicó que entrarían a una última fase -que se llevaría a cabo fuera del salón de clases pues el semestre había concluido- consistente en preparar todo el material para la entrega final y formal ante el cliente.

Durante esta fase final se ejecutaron los materiales en alta resolución para entregarlos a los respectivos impresores de los medios de comunicación en donde se implementaría la campaña. Además de afinar los detalles de la presentación con la que se expuso la propuesta al cliente, los alumnos explicaron - ante los ejecutivos de UNIFIN ${ }^{\circledR}$ - todo el proceso creativo que siguieron para generar la propuesta.

Ese mismo día la empresa realizó un video corporativo con la experiencia de los estudiantes, desde la explicación de sus ideas, recepción de su reconocimiento como ganadores $\mathrm{y}$, principalmente, la entrega de su premio: un automóvil para cada uno de los integrantes.

La campaña ganadora comprendió el siguiente principio creativo que compartimos para motivar a más alumnos de Diseño Gráfico a participar en este tipo de iniciativas por parte de las empresas corporativas y que vean las oportunidades que se pueden generar a partir de una clase semestral del último año:

- Campaña: "Armar"

- Desde niño has armado cosas. En el presente, nosotros te apoyamos para desarrollar, completar y concluir tus proyectos.

- Concepto creativo: "Juguete"

- Mensaje clave: UNIFIN ${ }^{\circledR}$, la pieza que completa tus proyectos.

- Justificación creativa: UNIFIN ${ }^{\circledR}$, comprometido con ofrecerte los mejores servicios, crece junto a tus mejores experiencias. Creemos que es tiempo de volverlas realidad y que las hagas parte de tu vida, por esta razón te brindamos todas las facilidades financieras para desarrollar, completar y concluir tus proyectos mediante nuestros ser- vicios de Leasing, Factoring, Crédito Automotriz e Insurance.

La experiencia se amplió al divulgarse el éxito de su campaña en publicaciones oficiales a nivel nacional, principalmente en los medios de difusión universitaria como la Gaceta UNAM y el NotiFES, el 27 de febrero del 2017.

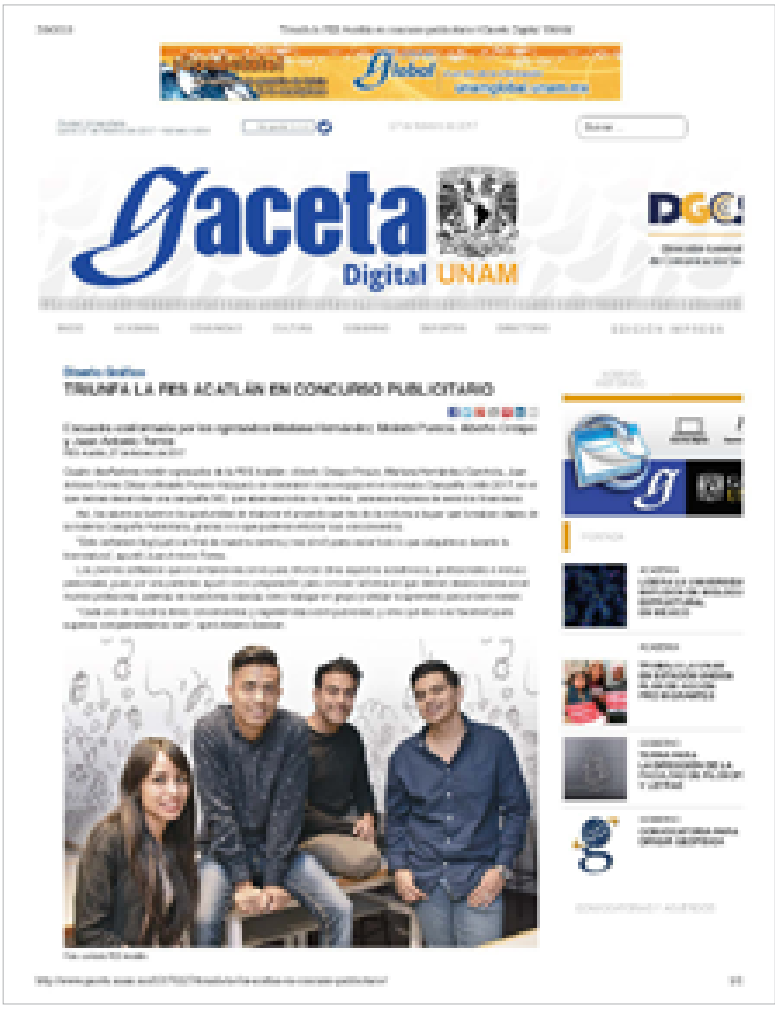

Figura 2. Gaceta Digital UNAM, 27 de febrero de 2017.

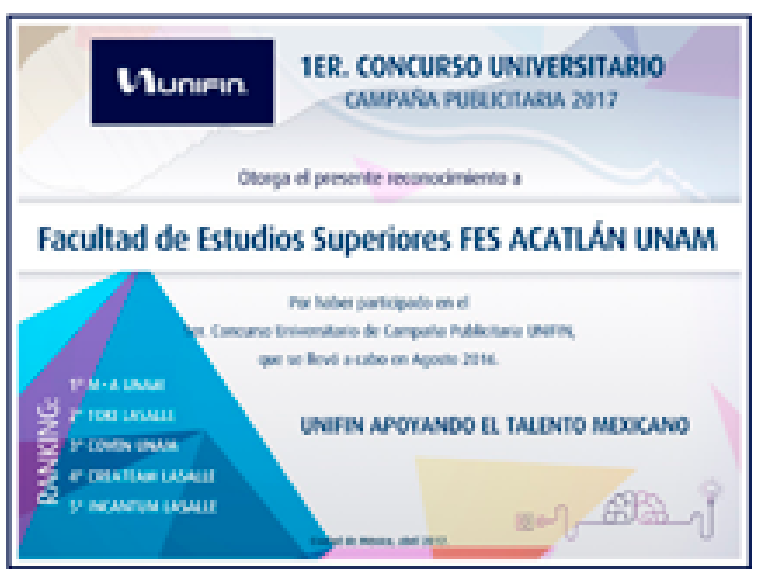

Figura 3. Diploma para la FES Acatlán, abril 2017 


\section{Conclusiones}

Este es otro gran ejemplo que merece ser compartido en nuestra comunidad de Diseño Gráfico y motivo de inspiración para seguir generando creatividad con soluciones efectivas a los requerimientos de nuestros clientes. El resultado obtenido invita a continuar impulsando el desarrollo de este tipo de proyectos de diseño de manera integral y multidisciplinaria, para concretar enfoques metodológicos y de investigación, con la contribución principal de la propia experiencia adquirida durante estos años en el ámbito creativo en diversas agencias de publicidad y diseño.

La materia de Campaña Publicitaria, impartida en $9^{\circ}$ semestre, ha sido una plataforma para diversas generaciones y servido para llevar a cabo proyectos reales con la oportunidad de vincularse al entorno laboral de la industria creativa, en donde los alumnos realizan aportaciones gráficas que se han implementado tanto en importantes instituciones o proyectos de incubación como en apoyo a instancias, tales como FES Acatlán, con los departamentos del Centro de Difusión Cultural; ${ }^{5}$ de Desarrollo Empresarial e Intercambio y Colaboración; ${ }^{6}$ en la CDMX, con el Museo Nacional de la Estampa, Zoológico de Chapultepec, Piso 16 y Jardín Botánico (los dos últimos ambos de la UNAM); empresas como Inroads ${ }^{\circledR}$, Teatro en corto, Kamedicis $^{\circledR}$, Atelier ${ }^{\circledR}$ Patrimonial. Marcas independientes: El Pestaquito, Muñecas “Tuti” ${ }^{\circledR}$, Mexaheads ${ }^{\circledR}$, Academia de Baile Roxana, Skal ${ }^{\circledR}$ International México, Helado Obscuro ${ }^{\circledR}$, Bissú ${ }^{\circledR}$ Cosméticos; así como algunas asociaciones civiles como ANSER, Red de mujeres A.C.A., Asociación $\mathrm{ALE}^{\circledR}$, Ballet Folklórico Mexiquense Acatlán, entre muchos otros proyectos que nos han apoyado brindando oportunidades de aprendizaje

5 Agradecimientos a la Dra. Lucía Elena Acosta Ugalde y a la Lic. Yuritzia Navarrete Ávila.

6 Lic. Fabiola Virginia Lara Treviño, y en su momento con la Lic. Claudia Sierra Magaña. para que los alumnos de Diseño Gráfico puedan transformar su creatividad en soluciones gráficas que funcionen para el entorno social.

Se agradece a cada una de ellas por todas las facilidades que nos han brindado para el cumplimiento de los objetivos de la materia y para sus propios proyectos empresariales de comunicación corporativa.

Aquí se puede consultar el evento "Diálogo de Saberes Multidisciplinarios" Temporada 1, organizado por el Sepedi (Seminario Permanente para la Divulgación de la Investigación) 2020, a cargo de la Dra. María del Rosario Hernández Coló; así mismo, se agradece el apoyo para la difusión y transmisión del evento a la Mtra. Xóchitl Hernández Martínez, coordinadora del programa de Enseñanza de Inglés de la División de Humanidades, FES Acatlán, UNAM. - 